\title{
El continente semántico de las representaciones: conocimiento compartido socialmente
}

\section{The semantic continent of representations: socially shared knowledge}

\section{(D)PSicoGENTE EISSN 2027-212X}

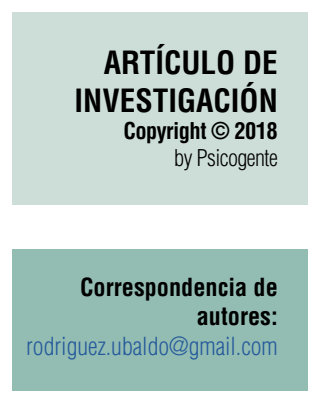

Recibido: $10-07-17$ Aceptado: 23-03-18 Publicado: 08-01-19

Ubaldo E. Rodríguez-De Ávila iD
Universidad del Magdalena, Santa Marta, Colombia

\section{Resumen}

Objetivo: Identificar el continente semántico de las representaciones sociales, partiendo de la hipótesis teórica de que "el conocimiento es socialmente compartido".

Método: El artículo presenta tres estudios en los que participaron un total de 1.659 sujetos, $52 \%$ hombres y $48 \%$ mujeres, seleccionados de manera intencional, por conveniencia, mayores de 16 años de edad, habitantes de la ciudad de Santa Marta, Colombia. Con metodología mixta y preponderancia en lo cualitativo, mediante las técnicas de Análisis Prototípico y Categorial de Representación Social, Análisis de Contenido y Lexicográfico, Asociación Libre de Palabras, apoyados con otros instrumentos, con 20 palabras o frases inductoras para extraer información.

Resultados: En los diferentes estudios se produjeron 5.983 tokens (palabras totales) y 1.806 types (palabras diferentes) y el promedio de types por sujeto fue de 1,3. El estudio demuestra que el conocimiento tiene Contenido, Organización y Significado concreto e individual, aunque compartido socialmente, y la estructura semántica que se moviliza conserva unidades significantes cuantitativamente comunes y reducidas.

Conclusión: Se demuestra que el continente semántico tiende a ser compacto, lo que sugiere que la cognición tiende a economizar energía y facilitar el comportamiento, yendo a interrelaciones más eficientes y estables.

Palabras clave: Representación social, Continente semántico, Análisis prototípico, Conocimiento compartido.

\section{Abstract}

Objective: This paper aims to identify semantic continet of social representations, focus on a theoretical hypothesis as follows: "socially shared knowledge".

Method: This paper shows three studies. 1659 participants were sampled, $52 \%$ men and $48 \%$ women, intentionally selected, by convenience, over 16 years of age, inhabitants in Santa Marta, Colombia. A mixed methodology showing preponderance of qualitative results; also, techniques of Prototypical and Categorical Analysis of Social Representation, Content Analysis and Lexicographic, Free Association of Words, supported with other instruments, 20 words or phrases inducing to extract information were used.

Results: According to different studies 5983 types (total words) and 1806 types (different words) and average of types per subject was 1.3. The study demonstrates that knowledge has concrete and individual although socially shared contents and the semantic structure that moved retains significant quantitatively common and reduced units.

Conclusion: It has been shown in this research that the semantic content tends to be compact, suggesting that cognition tends to save energy and facilitate behavior, addressed to more efficient and stable interrelationships.

Keywords: Social representation, Semantic continent, Prototypical analysis, Shared knowledge. 


\section{INTRODUCCIÓN}

En el año 2000 Falcon, según comentan Silva, Carmo y Silva (2015), planteó que representar significaba hacer presente una idea, algo o alguien, y Jovchelovitch (1998) en sus estudios apuntó que la expresión "Representaciones Sociales" fue mencionada por primera vez en 1961 por el rumano Serge Moscovici, quien negó la dicotomía existente entre lo individual y lo social, hasta entonces representada respectivamente por la Psicología que se encargaba de explicar al sujeto en su individualidad y por la sociología con sus estudios sobre la sociedad, en su colectivo, además de un sistema social de valores, ideas y prácticas (Diazgranados \& Sánchez, 2014). Así, resulta interesante verificar que las Representaciones Sociales de Moscovici es un modelo relativamente reciente, y vigente (Mora, 2002). De esta forma, en su estudio Psychanalyse: son imagen et son public, Moscovici (2013) dice que "representar significa a la vez y al mismo tiempo traer presente las cosas ausentes y presentar cosas de tal modo que satisfagan las condiciones de una coherencia argumentativa, de una racionalidad y de una integridad normativa del grupo" (p.216). Por lo tanto, es muy importante que esto se dé de forma comunicativa y difusiva, pues no hay otros medios, con la excepción del discurso y de los sentidos que contienen, por los cuales las personas y los grupos sean capaces de orientarse y adaptarse a las mismas (Moscovici, 2013).

Duveen (2013) por su parte, en la lógica de Moscovici, plantea que la Teoría de las Representaciones Sociales crea un puente entre los conceptos sociológicos y los psicológicos: “(...) la Teoría de las Representaciones Sociales de Moscovici buscó tanto reconocer un fenómeno social específico, como proporcionar los medios para hacerlo inteligible como un "Proceso sociopsicológico" (Duveen, 2013, p.28).

Moscovici $(1961 ; 1963 ; 1981 ; 1984)$ plantea conceptos importantes de las representaciones sociales, donde se centra en el constructo social como punto fundamental de muchos de los comportamientos humanos. Moscovici y Personnaz (1980) en dos experimentos clásicos, donde los efectos de los intentos de influencia por una mayoría y por una minoría fueron examinados tanto en un nivel de respuesta manifiesta como en un nivel perceptual latente; sujetos femeninos fueron expuestos a una serie de diapositivas azules consistentemente etiquetadas como verdes por una mujer presentada como miembro de una mayoría o una minoría; se predijo que "a) el juicio del sujeto de la imagen secundaria cromática sería modificado cuando el agente de influencia representara una minoría, y b) esta modificación será más pronunciada cuando la fuente de influencia está ausente que cuando está 
presente" (Moscovici \& Personnaz, 1980, p.270). Estos resultados apoyan la tesis que si dos personas independientes hacen la misma respuesta, el sujeto está obligado a atribuirla, al menos parcialmente, al propio estímulo, pero no podían descartar fácilmente su juicio atribuyéndolo a factores personales. De esta forma, Moscovici $(1990 ; 1998)$ ya planteó que se puede probar que la teoría de las representaciones sociales no propone nociones frívolas cuyo alcance es inmediatamente aparente. "Es un intento, tal vez prometedor, de pensar conjuntamente la explicación social, la clasificación y el sentido, la cognición y el discurso, y las operaciones y contenidos de las formas mentales (Moscovici, 1990, p.386).

En la teoría de las representaciones sociales no puede aislarse a la persona del contexto, principalmente desde el enfoque fundamental que comparten todas las ciencias sociales, en las cuales se desprende la idea de que todo conocimiento es socialmente compartido (Rodríguez, Cortés \& Varela, 2006; Rodríguez, 2006; 2009; 2011; Rodríguez \& Paba, 2013; Gómez, et al., 2011; Rodríguez, Espitia, Montenegro, Ortiz \& Suárez, 2014). De esta forma, el enfoque europeo de la representación social es un medio para socializar estos conceptos, pues se entiende que "la representación social se centra en la comunidad, las prácticas colectivas y la institucionalización del conocimiento social" (Farah, 2011, p.1593).

Así, estamos de acuerdo con lo que plantea Touraine (2007) respecto a lo que él llama "discours interprétatif dominant", lo cual dislocó la investigación en un tiempo específico para lugares exteriores al sujeto mismo en el análisis e interpretación de fenómenos sociales y de las conductas humanas precisamente sociales, relegando de forma despectiva a la "caja negra" todo lo que les pareciera lejano al abordaje metodológico de los análisis estadísticos y demostraciones de laboratorio. A pesar de todo, este movimiento, como plantea Jodelet (2015):

Permitió reintroducir la dimensión social en el abordaje de los fenómenos estudiados. Pero al mismo tiempo, esto llevó a la eliminación de la idea de sujeto como entidad psicológica y mental, volviendo la atención únicamente a los fenómenos de interacción y excluyendo todo un espacio ligado a la dinámica psíquica en la que se apoya la producción del pensamiento y de la acción (p.318).

Hoy día, el conocimiento que es socialmente compartido es tratado desde múltiples posibilidades, desde las nociones filosóficas de aprendizaje hasta los relatos de la Epistemología Social, en el que se presenta la "Cognición 
Socializada" (Knight \& Littleton, 2015; 2017; Knight, Arastoopour, Williamson, Buckingham \& Littleton, 2014) o cognición epistémica que es "socializada", poniendo como fundamento la naturaleza normativa y pragmática de las reivindicaciones de conocimiento, el contexto social en el que las demandas de saber ocurren a un nivel macro, incluyendo el contexto disciplinario y cultural, igualmente el contexto comunicativo en que se producen tales afirmaciones, las formas en que los individuos y los grupos pequeños expresan y construyen sus afirmaciones sobre el conocimiento. Es así como hay una "reorientación del análisis a nivel colectivo y las formas en que los estándares de conocimiento emergen de la actividad grupal, como propiedad comunicativa de esa actividad" (Knight \& Littleton, 2017, p.17).

Por otro lado, Kaës (1976) y Zavalloni (2007) trataron las relaciones entre representaciones sociales y subjetividad, y Moscovici (1984) incluye los "temas prioritarios que constituyen lo que se puede llamar objeto de una ciencia... el problema fundamental de la psicología social: el de la constitución del sujeto social" (p.66). Es precisamente ahí donde se ubica la premisa teórica de nuestro abordaje: En esa constitución del sujeto social, y no solo individual, ubicamos la naturaleza semántica y lexicográfica del conocimiento, el cual tiene Contenido, Organización y Significado concreto e individual, aunque compartido socialmente, y la estructura semántica que se moviliza conserva unidades significantes cuantitativamente comunes y reducidas (Rodríguez et al., 2006; Rodríguez, 2006; 2009; 2011; Rodríguez \& Paba, 2013; Gómez et al., 2011; Rodríguez et al., 2014).

El presente informe tiene como objetivo identificar el continente semántico de las representaciones sociales, partiendo de la hipótesis teórica de que "el conocimiento es socialmente compartido", como reconocimiento explícito de la complejidad del objeto de estudio: las Representaciones Sociales (RS), por cuanto se entiende como un fenómeno de tipo psicosocial. El recorrido metodológico fue cuidadosamente ejecutado para la premisa empírica de que una representación para ser elaborada socialmente necesita de la movilización de las estructuras cognitivas individuales, y por tanto los esquemas mentales (representación) que se proyectan en el comportamiento verbal deben configurarse de forma concreta en el continente semántico, en una especie de "economía cognitiva" reduciendo los significados a un bloque compacto y reducido de imágenes, símbolos o simplemente palabras.

Desde el año 2007 hemos elaborado esta idea que presentamos ahora para revelar de alguna forma cómo se compacta el continente semántico en la 
estructura de representaciones mentales de los sujetos, bajo los siguientes ejes: 1) Demostración de la hipótesis teórica de que el conocimiento es socialmente compartido, 2) El conocimiento socialmente compartido tiende a reducirse a un bloque semántico compacto que se evidencia en el comportamiento verbal, 3) Todo recorrido metodológico que apunte a verificar esta idea debe llevar a los mismos resultados.

\section{MÉTODO}

\subsection{Diseño}

El estudio es descriptivo tipo cualitativo, con técnicas cuantitativas en la presentación de los resultados. La unidad de análisis es el continente semántico y lexicográfico que movilizan las representaciones sociales de los sujetos (Jodelet, 2011; 2015; Knight et al., 2014; Knight \& Littleton, 2017). La "Representación Social" es entendida como un continente epistémico, un todo cognitivo, susceptible de ser segmentado en su análisis e interpretación (Ceirano, 2000; Durkheim, 1996; Jodelet, 1984; Silva et al., 2015), con características propias que la definen y que tienen un Contenido, una Organización y un Significado concreto e individual, aunque compartido socialmente, producidos por los sujetos en el discurso oral o escrito, o en su Referido Semántico, Conocimiento, Actitud, Percepción, Contenido, Organización y Significado, los que son precisamente segmentos medibles del "todo cognitivo" (Representación Social).

\subsection{Participantes}

El espectro muestral se constituyó de 1.659 sujetos (52\% hombres y $48 \%$ mujeres), con una selección de tipo intencional, por conveniencia, con el criterio ser residentes de la ciudad de Santa Marta, Colombia. El rango de edad fue de 16 a 60 años, sin embargo este criterio no se tuvo en cuenta como contraste o comparación, debido a los objetivos de la investigación.

\subsection{Instrumentos}

\subsubsection{Asociación Libre, Mapa Mental y Escala de Representación Social del Medio Ambiente}

Dos preguntas esenciales fueron presentadas: La primera permitió extraer información sobre el término <Centro Histórico de Santa Marta>, la segunda derivaba de la información suministrada por la primera: Nombrar los cinco lugares más representativos del Centro Histórico de Santa Marta, y enumerarlos en orden de importancia. Además se suministró una hoja en blanco 
para que los sujetos elaboraran un mapa mental de la ciudad de Santa Marta (Rodríguez et al., 2006; Rodríguez, 2009; Rodríguez \& Paba, 2013).

\subsubsection{Escala de Valoración Ambiental y dimensión afectiva}

Consistió en proponer los cinco lugares más representativos del Centro Histórico de Santa Marta extraídos del instrumento anterior, con 12 categorías de valor y una escala Likert de 1 a 5 , donde 1 era el valor más bajo y 5 el valor más alto, y se les pidió a los sujetos que valoraran los lugares teniendo en cuenta las categorías de valor y rango de la escala (Holahan, 2004; Rodríguez, 2009; 2011). Posteriormente, a los mismos lugares presentados, se les propuso cuatro dimensiones de afecto para que los sujetos escogieran entre las dimensiones con relación a cada lugar (Rodríguez, 2009; 2011). Conviene aclarar que los cuestionarios no fueron sometidos a un proceso de validación estadística debido a que por su naturaleza cualitativa no presentan homocedasticidad estadística.

\subsection{Procedimiento}

La investigación se desarrolló en tres estudios:

Estudio 1. Fue llevado a cabo en el año 2007. Participaron 210 sujetos, hombres (55\%) y mujeres (45\%). A 60 sujetos se les aplicó la técnica de mapas mentales; se les suministró una hoja de papel en blanco y lápiz negro y sin interferir, se les solicitó que dibujaran el Centro Histórico de la ciudad de Santa Marta, señalando los sitios representativos para ellos. A otros 60 sujetos diferentes se les aplicó la técnica de Asociación Libre (Pecly \& Wachelke, 2013) y se le pidió a cada sujeto que <sin pensarlo mucho> escribieran frases o palabras que le llegaran a la mente al escuchar la palabra inductora <Centro Histórico de Santa Marta>. Después de eso se les dijo que nombraran los cinco lugares más representativos (para ellos) del Centro Histórico de Santa Marta, y luego los enumeraran en orden de importancia (el valor 1 para el de mayor importancia y el valor 5 para el de menor importancia). La información de estos 120 sujetos fue procesada para definir los cinco lugares más representativos del Centro Histórico de Santa Marta, y luego, a un tercer grupo de 90 sujetos se les presentó la Escala de Valoración Ambiental con 12 categorías de valor con una escala Likert de 1 a 5 , donde 1 era el valor más bajo y 5 el valor más alto, y se les pidió que valoraran los lugares teniendo en cuenta las categorías de valor y rango de la escala. Posteriormente, a los mismos lugares presentados, se les propuso cuatro dimensiones de afecto para que los sujetos escogieran entre las dimensiones 
con relación a cada lugar. Esto con el fin de contrastar la información anterior. La premisa metodológica de este abordaje es el siguiente: Si el conocimiento es socialmente compartido, abordando la misma unidad de análisis (el lugar: Centro Histórico) en grupos diferentes, se deben extraer categorías semánticas y representaciones compactas o similares.

Estudio 2. Se realizó en el año 2010. La submuestra se constituyó de 212 sujetos, hombres ( $50 \%$ ) y mujeres ( $50 \%$ ). Las representaciones sociales fueron estudiadas a partir de la Escala de Representación Social del Medioambiente para 120 participantes. Y los que participaron del estudio de Asociación Libre del Medioambiente fueron 92 sujetos.

La palabra inductora en esta fase fue "Medio Ambiente". Para describir la Organización y el Contenido de la Representación Social del Medioambiente en los habitantes de Santa Marta, se tomaron los criterios de la técnica de Análisis Prototípico y Lexicográfico, también bajo la orientación teórica de la Teoría del Discurso.

Estudio 3. Se realizó durante el año 2015. La submuestra fue de 1.237 sujetos, hombres (48 \%) y mujeres (52\%). Se dividieron en 18 subgrupos, en los que se usaron diferentes palabras inductoras de la Representación Social a partir de la técnica de Asociación Libre, tomadas al azar, con el fin de verificar que el continente semántico se moviliza lexicográficamente de forma compacta y compartida. Y la técnica de recolección de información fue Asociación Libre.

\subsection{Análisis de datos}

La exploración de la RS se fundamentó en lo que hemos definido como "Análisis Prototípico y Categorial de Representación Social", desarrollado por el profesor Pierre Vergès (Navarro \& Gaviria, 2010), a partir de una tarea de Asociación Libre de Palabras, evocadas a partir de palabras inductoras específicas. La hipótesis de partida de esta técnica de recolección de información es la existencia de un funcionamiento cognitivo a partir del cual algunos términos son inmediatamente movilizados para expresar una representación (Navarro, 2004; 2006). Luego es constatado con la Escala de Valoración Ambiental (Holahan, 2004). El fundamento teórico fue el análisis de contenido (López-Noguero, 2002; Kuri-Pineda, 2017), apoyado por la Teoría del Discurso (Piñuel, 2002) vamos a mostrar que en un discurso extraído por medio de esta técnica hay reglas de formación de objetos (que no son las reglas de utilización de las palabras), reglas de formación de conceptos (que no son las leyes de la sintaxis) y reglas de formación de teorías (que no son ni 
deductivas ni retóricas). Posteriormente se sometió a un análisis descriptivo estadístico por medio del programa SPSS versión 15.

\subsection{Consideraciones éticas}

Se declara que no existió ningún conflicto ético durante la realización del estudio, el cual contó con la aprobación de un comité de ética en investigación. Los participantes se encontraban de acuerdon en participar del estudio, el cual estuvo en consonancia con la Resolución 8430 que reglamentó la investigación en salud (Ministerio de Salud, 1993).

\section{RESULTADOS}

\subsection{Estudio 1}

En la fase de Asociación Libre, en las que participaron 60 sujetos, se produjeron 245 palabras o expresiones de las cuales 113 son palabras diferentes, es decir, un promedio de 4 palabras por sujeto y 1,9 palabras diferentes por persona. A partir de este primer corpus se realizó un análisis del prototipo de la Representación Social (RS) del Centro Histórico de Santa Marta (CH-SM).

Tabla 1.

Prototipo (rango X frecuencia) de la RS del CH-SM

\begin{tabular}{|c|c|c|}
\hline & $<=2$ & RANGO MEDIO >2 \\
\hline$>=10$ & Historia $23 * 1,957 * *$ & $\begin{array}{c}\text { Antigüedad } 16^{*} 2,125^{* *} \\
\text { Cultura } 14^{*} 2,214^{* *} \\
\text { Catedral } 12 * 2,250^{* *}\end{array}$ \\
\hline $\begin{array}{l}\text { Frecuencia } \\
\quad<10\end{array}$ & & $\begin{array}{c}\text { Monumentos } 7 * 2,571^{* *} \\
\text { Casas Antiguas } 7 * 2,429 * * \\
\text { Claustro San Juan } \\
\text { Nepomuceno } 5 * 3,600^{* *} \\
\text { Antepasado } 5 * 3,800^{* *}\end{array}$ \\
\hline
\end{tabular}

*Frecuencia de evocación

**Rango promedio del ítem.

Se obtuvo luego del análisis de prototipicidad un segundo corpus de 8 palabras (ver Tabla 1). En primer lugar se descubrió que el núcleo central está formado por la palabra HISTORIA. Esta expresaría una condición general de la manera en que los sujetos perciben este lugar. En el periférico 1 (columna superior derecha de la Tabla 1) se encontraron otras denominaciones utilizadas para nombrar y categorizar al CH-SM. Son denominaciones que expresan la diversidad de percepciones que hacen referencia a este lugar: Una se refiere a la Antigüedad, otra a la condición de lugar (Catedral) y la otra a la Cultura. 
A partir de esta interpretación, un análisis de categorías es propuesto para conocer los temas que componen la RS del CH-SM. Tres categorías surgieron inicialmente del análisis prototípico (ver Tabla 2) organizando el sentido de las 8 palabras del prototipo.

Tabla 2.

Categorías presentes en el prototipo de la RS del CH-SM

\begin{tabular}{ccc}
\hline LUGAR & HISTORIA & CULTURA \\
Catedral & Historia & Cultura \\
Claustro San Juan Nepomuceno & Antigüedad & Monumentos \\
& Antepasado & Casas Antiguas \\
\hline
\end{tabular}

Dos lugares fueron evocados con fuerza y pertenecen al segundo corpus del prototipo (ver Tabla 1). El resto son palabras que se organizan alrededor de la palabra que constituye el núcleo central del prototipo: HISTORIA. En la categoría "Lugar" los individuos reconocen con intensidad representacional la Catedral y el Claustro San Juan Nepomuceno. Esto se confirma cuando analizamos las dos categorías que siguen: "Historia" y "Cultura", las palabras Historia, Antigüedad, Antepasado, Cultura, Monumentos y Casas Antiguas son evocadas con fuerza organizando la RS del CH-SM en la construcción de la identidad social urbana del habitante de Santa Marta.

Además de las tres categorías extraídas del prototipo, surgieron siete categorías más que hacen referencia a la percepción del $\mathrm{CH}-\mathrm{SM}$ como un fenómeno de turismo (playas, vacaciones, diversión, etc.), plagado de amor, valor, importancia, en la categoría de "Valores/sentimientos". También surgió la categoría "denominación social" que menciona los sujetos usuarios del CH-SM en la relación de estos en la vida cotidiana (vendedores, multitud, gente, gamín, etc.). Otra categoría fue "organización/seguridad", plasmada de forma negativa como robo, abandono, descuido, desorden, etc. Otra de las categorías fue "socio-política", nombrándose aquí política, pobreza, poco progreso y falta de gestión, estableciéndose por extensión al sentido perceptual de los sujetos estudiados y los procesos político-administrativos de la ciudad. Otra categoría resultante fue "comercio", indicando aquí compras, el comercio mismo, comprar y vendedores ambulantes. La última categoría elaborada fue "abstracción-realidad", donde los sujetos nombran las palabras imaginación o realidad para referirse al objeto de estudio (Tabla 3).

La categoría que más palabras tiene, y por extensión con más riqueza semántica es "Iugares" con un 29,6 \% sobre el total de palabras diferentes evocadas, 
seguida en su orden por las categorías "historia" (15,8\%), "cultura" (11,5\%), y "Valores/sentimientos" (11,5\%). En cuanto al porcentaje de evocaciones permanece el orden, donde a la categoría "lugar" le corresponde el 36,5 \% del total de las evocaciones, seguida de la categoría "historia" (28,5\%).

Tabla 3.

Análisis Categorial: jerarquía entre las categorías de la RS del CH-SM

\begin{tabular}{ccccc}
\hline CATEGORÍA & $\begin{array}{c}\text { NO. DE } \\
\text { PALABRAS }\end{array}$ & $\%$ & $\begin{array}{c}\text { OCURRENCIAS (NO. } \\
\text { DE EVOCACIONES) }\end{array}$ & $\%$ \\
\hline 1. Lugares & 33 & 29,6 & 89 & 36,5 \\
2. Historia & 18 & 15,8 & 70 & 28,5 \\
3. Cultura & 13 & 11,5 & 23 & 9,3 \\
4. Valores/sentimientos & 13 & 11,5 & 17 & 6,9 \\
5. Turismo & 10 & 8,8 & 13 & 5,5 \\
6. Denominación social & 8 & 7 & 11 & 4,4 \\
7. Organización/seguridad & 8 & 7 & 10 & 4 \\
8. Socio-política & 4 & 3,5 & 4 & 1,7 \\
9. Comercio & 4 & 3,5 & 6 & 2,4 \\
10. Abstracción- realidad & 2 & 1,8 & 2 & 0,8 \\
TOTAL & 113 & 100 & 245 & 100 \\
\hline
\end{tabular}

En cuanto al contenido de la representación socioespacial se encontró lo siguiente: Con un total de 292 evocaciones, 44 lugares se repitieron, donde la catedral, la Plaza de Bolívar, el Camellón, el Museo del Oro, la Alcaldía y el Claustro San Juan Nepomuceno, con porcentajes del 15 \%, 12 \%, 8\%, 7\%, 6 \% y $5 \%$ respectivamente, son los más representativos. Y en cuanto a la organización de la RSE-CHS, el tipo de Mapas Mentales en los sujetos estudiados tuvieron una distribución del $47 \%$ para el tipo Secuencial; el $43 \%$ para el tipo Espacial y el $10 \%$ fueron considerados No Mapas. Con 590 lugares dibujados en los Mapas Mentales, 144 fueron lugares diferentes, y los que tienen mayor carga identitaria más o menos pronunciada para los sujetos, si tenemos en cuenta una distribución mayor o igual al 20 \% sobre ese total, se destacan dentro de la imagen colectiva la Plaza Simón Bolívar, la Catedral y el Camellón con una distribución superior al $40 \%$.

La Catedral fue valorada por el $66 \%$ de los sujetos con valor de 5 en su organización, la Plaza Simón Bolívar, en un 31 \% en valor 5, el Camellón en un $43 \%$ y al Museo del Oro el $84 \%$ de las personas estudiadas lo valoran con puntaje de 5 en la organización. Y al Claustro San Juan Nepomuceno, el 61 \% lo valoraron en 5 . Por otro lado, la valoración afectiva o vínculo identitario entre sujeto-lugar, en orden de valoración se encuentran la Catedral, la 
Plaza Simón Bolívar, el Camellón, el Museo del Oro y el Claustro San Juan Nepomuceno.

\subsection{Estudio 2}

En lo que respecta al Contenido, Organización y Significado de Medio Ambiente en los habitantes de Santa Marta, con 92 sujetos participantes en esta fase de Asociación Libre, en el estudio se produjeron 513 palabras o expresiones (5,5 expresiones por sujeto) de las cuales 165 son palabras diferentes, es decir, un promedio de 1,7 palabras diferentes por persona.

A partir de este primer corpus se realizó un análisis del prototipo de la RS del Medioambiente, con el fin de reconocer la jerarquía de los elementos que la componen y su organización estructural, permitiendo comprender el sentido particular que los sujetos sociales tienen de la RS del Medio Ambiente. Se extrae la coocurrencia de las palabras más significativas de las evocaciones de los sujetos, que corresponden al $63 \%$ de las evocaciones totales (ver Tabla 4), ordenadas alfabéticamente.

\section{Tabla 4.}

Coocurrencias de Palabras Evocadas

\begin{tabular}{ccc}
\hline 1: AGUA & 11: ECOSISTEMA & 21: RÍOS \\
2: AIRE & 12: ENTORNO & 22: SALUD \\
3: ANIMALES & 13: FAUNA & 23: SELVA \\
4: ÁRBOLES & 14: FLORA & 24: SERES-HUMANOS \\
5: ATMÓSFERA & 15: HOMBRE & 25: SERES-VIVOS \\
6: BIOSFERA & 16: MARES & 26: SUELO \\
7: BOSQUES & 17: MONTAÑAS & 27: TIERRA \\
8: CAPA-DE-OZONO & 18: NATURALEZA & 28: VEGETACIÓN \\
9: CONTAMINACIÓN & 19: OXÍGENO & 29: VERDE \\
10: CULTURA & 20: PLANTAS & 30: VIDA \\
\hline
\end{tabular}

Se obtuvo luego el análisis de prototipicidad, un segundo corpus de 23 palabras (Tabla 5), las más importantes para referirse al objeto de representación.

Tabla 5.

Prototipo (rango X frecuencia) de la RS del Medioambiente.

\begin{tabular}{ccc}
\hline & \multicolumn{2}{c}{ RANGO PROMEDIO } \\
\hline & $<3,5$ & $\geq 3,5$ \\
& NATURALEZA 52 2,365 & MARES 20 3,550 \\
& AGUA 24 3,167 & SERES-VIVOS 20 4,050 \\
& AIRE 23 2,957 & RíOS 17 3,647 \\
& ANIMALES 23 3,261 & BOSOUES 15 4,733 \\
& CONTAMINACIÓN 21 3,429 & FAUNA 11 4,364
\end{tabular}




\begin{tabular}{|c|c|c|}
\hline \multicolumn{3}{|c|}{ RANGO PROMEDIO } \\
\hline \multirow{4}{*}{ Frecuencia } & ÁRBOLES 162,813 & \\
\hline & TIERRA 12 2,417 & \\
\hline & SUELO 5 2,800 & FLORA 93,778 \\
\hline & SALUD 5 3,200 & VIDA 94,000 \\
\hline \multirow{7}{*}{$>5$} & & CAPA-DE-OZONO 83,875 \\
\hline & & PLANTAS 63,500 \\
\hline & & ENTORNO 63,667 \\
\hline & & OXÍGENO 64,333 \\
\hline & & ATMÓSFERA 5 4,000 \\
\hline & & VEGETACIÓN 5 4,000 \\
\hline & & SERES-HUMANOS 5 5,200 \\
\hline
\end{tabular}

En primer lugar se descubrió que el núcleo central está formado por las palabras NATURALEZA, AGUA, AIRE, ANIMALES, CONTAMINACIÓN, ÁRBOLES y TIERRA, haciendo referencia a la denominación que comúnmente se usa para nombrar al Medioambiente. Estas palabras expresan una condición general de la manera en que los sujetos perciben al Medioambiente (MA).

A partir de esta interpretación útil y necesaria, un análisis de categorías es propuesto para conocer los temas que componen la RS del MA y que ayudan a identificar además del Contenido, toda la Organización de la RS del MA. Tres categorías surgieron inicialmente del análisis prototípico (ver Tabla 6) organizando el sentido de las 23 palabras del prototipo.

Tabla 6.

Categorías presentes en el prototipo de la RS del MA

\begin{tabular}{ccc}
\hline NATURALEZA & GLOBALIDAD & VIDA \\
\hline TIERRA & ENTORNO & AGUA \\
MARES & CONTAMINACIÓN & AIRE \\
RÍOS & SALUD & OXÍGENO \\
BOSQUES & & SUELO \\
NATURALEZA & CAPA DE OZONO \\
ANIMALES & ATMÓSFERA \\
SERES-VIVOS & VIDA \\
PLANTAS &
\end{tabular}




\begin{tabular}{cc}
\hline NATURALEZA & GLOBALIDAD \\
\hline ÁRBOLES & \\
SERES-HUMANOS & \\
FAUNA & \\
FLORA & \\
VEGETACIÓN & \\
\hline
\end{tabular}

A partir de las tres categorías extraídas del prototipo, resultaron cinco definitivas que hacen referencia al significado del Medioambiente, su contenido y organización (ver Tabla 7) como un fenómeno de recursos naturales de la biosfera (ecosistema, mares, ríos, agua, capa de ozono, tierra, etc.), al mismo tiempo como degradación, contaminación, enfermedad, tala de árboles, sociedad, pertinencia, práctica cultural, etc., en la categoría de "contaminación, sociedad, cultura" (ver Tabla 6) se menciona la relación de los sujetos estudiados con el Medioambiente, como cuidado de la naturaleza, preservación. Otra categoría fue "Entorno y Medio Físico", concibiéndose el Ambiente como lo relacionado con la ciudad y el entorno espacial donde se desenvuelven las personas.

Al mismo tiempo, ahondando en el tema del significado del Medioambiente propiamente dicho, a través de un abordaje cualitativo, se identificaron cinco categorías de significados del Medioambiente entre las diferentes respuestas dadas por los sujetos. 86 sujetos dieron significados y la categoría con mayor riqueza semántica es el Entorno en que se desenvuelve el hombre con el $45 \%$ de representatividad y 39 significados, de los cuales se pueden extraer frases como: "Entorno", "todo aquello que nos rodea", "entorno en que vivimos que influye tanto física, psicológica, social y culturalmente al hombre", "entorno que afecta y condiciona especialmente las circunstancias de vida de las personas y sociedad en su conjunto", "todo lo que nos rodea por eso debemos conservarlo y cuidarlo para así seguir disfrutando". En la categoría Naturaleza con 22 significantes y un porcentaje de representatividad del $26 \%$, encontramos frases como: "naturaleza en General", "naturaleza", "conjunto de elementos abióticos y bióticos que integran la capa llamada biosfera". En la categoría Espacio o Lugar, con un significante de 11 y un porcentaje del $13 \%$ se encontraron frases como: "espacio que convivimos con los demás seres vivos", "lugar en que se encuentran todos los seres vivos". 
Tabla 7.

Categorías asociadas al análisis lexicográfico de la RS del MA

\begin{tabular}{ccc}
\hline CATEgORÍAS & $\begin{array}{c}\text { OCURRENCIAS (NO. } \\
\text { EVOCACIONES) }\end{array}$ & $\begin{array}{c}\% \text { DE } \\
\text { OCURRENCIAS }\end{array}$ \\
\hline 1-Naturaleza & 206 & $40 \%$ \\
2-Recursos Naturales de la Biosfera & 135 & $26 \%$ \\
3-Contaminación, Sociedad y Cultura & 114 & $22 \%$ \\
4-Entorno y Medio Físico & 25 & $5 \%$ \\
5-Vida & 33 & $6 \%$ \\
TOTAL & 513 & $100 \%$ \\
\hline
\end{tabular}

En la categoría de Prácticas Negativas, con un significante de 7 frases (8\%), se encontraron los siguientes significados: "contaminación de la tierra y agua", "Falta de sentido de pertenencia", "falta de cuidado con el medioambiente". Y en la categoría Vida, con 7 significantes ( $8 \%$ ), se encontraron las siguientes frases: "Vida", "Salud y cuidado ambiental".

\subsection{Estudio 3}

Después de encontrar en los estudios 1 y 2 una estructura lexicográfica (o continente semántico) que apoya la tesis fundamental del presente informe, se decidió abordarlo con una variante particular: Se tomaron 18 grupos de personas, con un total de 1.237 participantes, a quienes se les pidió escribieran lo que primero se les viniera a la mente al presentárseles la respectiva frase inductora (ver Tabla 8). No se analizó el discurso propiamente dicho, no buscábamos los significados en sí mismos, más bien comprobar nuestra hipótesis de partida, con la técnica de Asociación Libre (Pecly \& Wachelke, 2013).

Tabla 8.

Grupos de sujetos y palabras o frases inductoras en el estudio de Asociación Libre

\begin{tabular}{ccccc}
\hline \multirow{2}{*}{ PALABRA O FRASE INDUCTORA } & \multirow{N}{*}{} & \multirow{2}{*}{ POBLACIÓN } & \multicolumn{2}{c}{ GÉNERO } \\
\cline { 4 - 5 } & & & HOMBRES & MUJERES \\
\hline BARRAS BRAVAS & 60 & HINCHAS DE FÚTBOL & 58 & 2 \\
AMOR & 60 & ESTUDIANTES & 23 & 37 \\
POBREZA & 60 & HABITANTES POBRES & 18 & 42 \\
DOLOR CRÓNICO & 60 & PACIENTES & 26 & 34 \\
BULLYING & 60 & ESTUDIANTES & 43 & 27 \\
RENDIMIENTO ACADÉMICO & 60 & ESTUDIANTES & 28 & 32 \\
ESTRATEGIAS DE APRENDIZAJE & 60 & DOCENTES & 12 & 48
\end{tabular}




\begin{tabular}{ccccc}
\hline & & & & \\
& & & & \\
CÁNCER DE CUELLO UTERINO & 60 & ESTUDIANTES & 0 & 60 \\
AUTOESTIMA & 60 & ESTUDIANTES & 30 & 30 \\
VIOLENCIA ESCOLAR & 60 & ESTUDIANTES & 31 & 29 \\
ESTRÉS ACADÉMICO & 70 & ESTUDIANTES & 35 & 35 \\
MÉTODOS ANTICONCEPTIVOS & 76 & ESTUDIANTES & 37 & 39 \\
FUNCIONES EJECUTIVAS & 60 & ESTUDIANTES & 18 & 42 \\
DELITO & 70 & PRESIDIARIAS & 38 & 22 \\
ORIENTACIÓN VOCACIONAL & 70 & ESTUDIANTES & 17 & 53 \\
ASESINOS EN SERIE & 167 & ESTUDIANTES & 100 & 67 \\
TÉCNICA ABA & 64 & DOCENTES & 40 & 24 \\
EMOCIONALIDAD & 60 & PADRES & 40 & 20 \\
& 1237 & & 594 & 643 \\
\hline
\end{tabular}

El estudio arrojó 5.225 palabras en total, de las cuales 1.528 eran palabras diferentes. El promedio de palabras totales por sujeto fue de 4,22 palabras y el promedio de palabras diferentes por sujeto fue de 1,24 (ver Tabla 9).

Tabla 9.

Corpus del contenido de las representaciones sociales

\begin{tabular}{ccccc}
\hline PALABRA O FRASE INDUCTORA & PALABRAS TOTALES & $\begin{array}{c}\text { PALABRAS } \\
\text { DIFERENTES }\end{array}$ & $\begin{array}{c}\text { PROM. PALA- } \\
\text { BRAS TOTALES } \\
\text { POR SUJETO }\end{array}$ & $\begin{array}{c}\text { PROM. PALABRAS } \\
\text { DIFERENTES POR } \\
\text { SUJETO }\end{array}$ \\
\hline BARRAS BRAVAS & 283 & 56 & 4,72 & 0,93 \\
AMOR & 298 & 81 & 4,97 & 1,35 \\
POBREZA & 238 & 66 & 3,97 & 1,10 \\
DOLOR CRÓNICO & 262 & 45 & 4,37 & 0,75 \\
BULLYING & 229 & 57 & 3,82 & 0,95 \\
RENDIMIENTO ACADÉMICO & 298 & 68 & 4,97 & 1,13 \\
ESTRATEGIAS DE APRENDIZAJE & 288 & 35 & 4,8 & 0,58 \\
CÁNCER DE CUELLO UTERINO & 300 & 38 & 5 & 0,63 \\
AUTOESTIMA & 246 & 63 & 4,1 & 1,05 \\
VIOLENCIA ESCOLAR & 209 & 73 & 3,48 & 1,22 \\
ESTRÉS ACADÉMICO & 350 & 97 & 5 & 1,39 \\
METODOS ANTICONCEPTIVOS & 373 & 59 & 4,91 & 0,78 \\
FUNCIONES EJECUTIVAS & 281 & 122 & 4,68 & 2,03 \\
DELITO & 326 & 125 & 4,66 & 1,79 \\
ORIENTACIÓN VOCACIONAL & 320 & 152 & 4,57 & 2,17 \\
ASESINOS EN SERIE & 384 & 202 & 2,30 & 1,21 \\
TECNICA ABA & 256 & 98 & 4 & 1,53 \\
EMOCIONALIDAD & 284 & 91 & 4,73 & 1,52 \\
& 5225 & 1528 & 4,22 & 1,24 \\
\hline
\end{tabular}




\section{DISCUSIÓN}

De acuerdo con la propuesta de Pecly y Wachelke (2013), a partir de una tarea de evocación, es posible hacer una gran cantidad de análisis; la mayoría de ellos se interesan por el contenido evocado y no por la organización, y mucho menos por la naturaleza compacta del bloque energético que alimentan los significados dentro de la estructura mental que da vida a las representaciones. Con los datos aquí presentados (ver Tabla 10), se demuestra que el promedio de palabras diferentes por sujeto (types) es de 1,24, es decir, que el bloque significante se concreta o se reduce a una expresión de contenido. Podemos deducir que la complejidad de los significados obedece a lógicas independientes de la dinámica social, aunque se fundamente en ella.

De acuerdo con Navarro y Gaviria (2010), la categorización que se hace en este tipo de estudio se ve como una necesidad de reducir la complejidad y darle forma a lo que se conoce como a lo que se desconoce, creando una visión coherente y clara de la realidad social para asimilarla. Se sabe también que este proceso de categorización de las personas se hace a través de una simplificación, de una reducción abusiva de las características del objeto que va a permitir y justificar las generalizaciones (ver Tabla 10).

Tabla 10.

Corpus del contenido de las representaciones sociales por estudio

\begin{tabular}{ccccccc}
\hline & $\begin{array}{c}\text { PALABRAS } \\
\text { OFRASES } \\
\text { INDUCTORAS }\end{array}$ & $\begin{array}{c}\text { PARTICIPAN- } \\
\text { TES }\end{array}$ & $\begin{array}{c}\text { PALA- } \\
\text { BRAS } \\
\text { EVOCA- } \\
\text { DAS }\end{array}$ & $\begin{array}{c}\text { PALABRAS } \\
\text { DIFEREN- } \\
\text { TES }\end{array}$ & $\begin{array}{c}\text { PROMEDIO } \\
\text { PALABRAS } \\
\text { EVOCADAS } \\
\text { POR SUJETO }\end{array}$ & $\begin{array}{c}\text { PRO- } \\
\text { MEDIO } \\
\text { PALABRAS } \\
\text { DIFEREN- } \\
\text { TES POR } \\
\text { SUJETO }\end{array}$ \\
\hline ESTUDIO 1 & 1 & 60 & 245 & 113 & 4 & 1,9 \\
ESTUDIO 2 & 1 & 92 & 513 & 165 & 5,5 & 1,7 \\
ESTUDIO 3 & 18 & 1237 & 5225 & 1528 & 4,22 & 1,2 \\
TOTALES & 20 & 1389 & 5983 & 1806 & 4,31 & 1,3 \\
\hline
\end{tabular}

Nota: La muestra total del estudio fue de 1659. La diferencia de 270 sujetos que se visualiza en esta tabla, corresponde a 150 sujetos del estudio 1 y 120 sujetos del estudio 2, que se usaron como verificación empírica de la organización y contenido de la RS (ver ítem metodología)

Analizando el contenido de la RSE del CH-SM, se debe partir del reconocimiento de los lugares como eje central en la construcción de la identidad social, tomados de la RS que los sujetos tienen compuesto, organizado y estructurado en su relación con el lugar. Esto se explica por el principio de organización de los elementos que componen una experiencia perceptiva y que los gestaltistas Ilamaron Pregnancia (Prägnanz). O uno de los esquemas 
que permiten organizar la información proveniente del entorno: "proximidad" (Rodríguez et al., 2006; Rodríguez, 2006; 2009), pues las distancias cortas o los objetos cercanos en el espacio urbano, contribuyen a la percepción de grupos unitarios, es decir, permite a las personas relacionar elementos que se encuentran relativamente cerca, lo cual lleva a pensar que ciertos lugares serán tenidos en cuenta con mayor prevalencia por encontrarse cerca a lugares representativos, más que por poseer características o patrones unificados. Es decir, se encuentran cerca de lugares legibles. A partir de los planteamientos del Interaccionismo Simbólico, se considera al "lugar" como el escenario donde los grupos sociales interactúan y el entorno es un elemento más de la interacción, ya que este al ser dotado de significado, es un producto socialmente elaborado a través de la interacción simbólica (Blumer, 1982).

Desde el análisis del contenido de la RS del Medioambiente, se debe partir del reconocimiento del entorno como eje central en la construcción de la identidad social frente al problema ambiental, tomado de la RS que los sujetos tienen compuesto, organizado y estructurado en su relación con el ambiente. Una frase, que se convierte en categoría y significante, con gran fuerza semántica para la construcción de la relación sujeto-ambiente, fue de gran valía en el estudio. De todo esto, a partir de la comprensión teórica de las RS como eje fundamental en Psicología Social, Psicología Ambiental y Educación Ambiental, puede entenderse como el producto de procesos psicológicos perceptuales y cognitivos mediante los cuales las personas comprenden, organizan e interactúan con el medio que les rodea, dándole sentido y significado (De Alba, 2004; Flores, 2008; 2010; González, 2001; Márquez-Pulido, 2014; Reigota, 1995; Mendlovic-Pasol, 2014; Merlinsky, 2017).

Interesantemente, los estudios de las representaciones sociales se estructuran con objetivos de conocer significados y significantes particulares, segmentados en razas, fajas etarias, regiones, culturas y tiempos, y en ese objetivo parecen desviarse del principio del concepto fundamental del constructo representación social (el conocimiento es socialmente construido y por tanto socialmente compartido). Es posible tener informaciones sobre la totalidad del corpus, y esas informaciones permiten una mejor comprensión de la representación social estudiada. El objetivo aquí no es proponer que el estudio del contenido representacional sea abandonado, sino atribuir mayor atención a las características del corpus representacional. 
Aunque esta no es una crítica a la Psicología de las Representaciones Sociales, ni a la Psicología Social misma, parece ser que la influencia social, las actitudes, los significados, etc., como variables propias, unidas o separadas, de las representaciones sociales, son contradictorias con la premisa misma en la cual se fundan. $Y$ tal parece que el mismo Moscovici posibilitó en sus seguidores dicha confusión teórica llevando un sesgo metodológico cuando se analizan las realidades que se sitúan en la intersección de lo social y lo psicológico. ¿Cuándo un fenómeno social puede considerarse psicológico y cuando un fenómeno psicológico puede considerarse social?, ¿cuándo una variable psicosocial está situada precisamente en esa intersección PsicoSocial?, son preguntas que no necesariamente deben resolverse en estos momentos, como tampoco fueron preguntas que consideramos al comenzar el presente estudio. Son cuestionamientos que surgen como discusión para sustentar el hecho de que el conocimiento debe seguir su camino y los que la construyen deben tener en mente todas las consideraciones planteadas.

Como plantea Jodelet (2011) acompañado igualmente por Silva et al. (2015), uno de los motivos que más llevó a Moscovici a desarrollar el estudio de las representaciones sociales, dentro de una metodología científica, fue su crítica a los presupuestos positivistas y funcionalistas de las demás teorías que no explicaban la realidad bajo diversas dimensiones. En esa lógica, Jodelet (2011) entiende a Moscovici (2013) en estos términos (y debemos reconocer que Denise Jodelet es una de las autoras que quizá más lo han comprendido):

Entre las razones que explican la fuerza de ese movimiento, ya mencioné el alcance de la Teoría de las Representaciones Sociales. La diversidad de las dimensiones psíquicas, intelectuales y cognitivas que ella abraza, la diversidad de los niveles sociales, individuales, interindividuales, intergrupales e ideológicos que ella articula permiten dar cuenta de la complejidad de los fenómenos que una psicología auténticamente social debe considerar (Jodelet, 2011, p.21).

Entender la complejidad pluridimensional de la realidad diversa no debe significar que no existen leyes que regulen tal complejidad. Los investigadores de las Representaciones Sociales reducen el abordaje metodológico, pero analizan los resultados como si en esa reducción estuviera la complejidad misma de la realidad diversa, luego no se atreven a generalizar sus hallazgos porque en el fondo quizá están alejados de las leyes que regulan los fenómenos que observan. Sin proponer un nuevo reduccionismo para la Psicología Social y el estudio de las Representaciones, creemos que las 
futuras investigaciones en esta área deben tener en cuenta el sesgo teórico que muchas publicaciones ponen de manifiesto.

Es constante de las ciencias sociales formar categorías ricas en conceptos, con la idea de que ayudan a las personas a navegar por el complejo mundo social al permitirles razonar sobre los pensamientos, creencias, acciones e interacciones de los demás, guiados por la pertenencia a grupos (Liberman, Woodward \& Kinzler, 2017; Zamora-Saenz, 2017) que, sin esa categorización social, a menudo tendrían consecuencias nefastas. Minimizar la categorización para comprender la realidad de manera sencilla debe ser el papel del científico. En el presente estudio se demuestra que el continente semántico tiende a ser compacto, lo que sugiere la hipótesis que la cognición tiende a economizar energía y facilitar el comportamiento, yendo a relaciones más eficientes y estables; que derivan de un proceso evolutivo de la cognición humana (Bar-Yosef, 2017) hacia adaptaciones funcionales a un entorno de relaciones (Suchow, Bourgin \& Griffiths, 2017) por amenazas potenciales como factor crítico de supervivencia (Debiec \& Olsson, 2017). De ahí que la capacidad de formar categorías sociales abstractas puede ser separable de las consecuencias negativas de la categorización social, incluyendo el prejuicio, la discriminación y los estereotipos (Liberman et al., 2017).

Financiamiento: El presente informe hace parte de la actividad investigativa realizada por el autor en el marco de la Tesis de Maestría en Educación del Sistema de Universidades Estatales del Caribe colombiano -SUE Caribe, sede Universidad del Magdalena

Agradecimientos: Al PhD Óscar Narro Carrascal, por su direccionamiento en Psicología Social y Ambiental; a la PhD Carmelina Paba Barbosa por su asesoría científica; a las psicólogas Sharol Cortés y Maira Varela, por su ayuda y compromiso en parte de las investigaciones realizadas en el área.

\section{REFERENCIAS}

Bar-Yosef, O. (2017). Can archaeology tell us about the evolution of cognition and language? Journal of Neurolinguistics, 43, 222-227. https://doi.org/10.1016/j. jneuroling.2016.11.009

Blumer, H. (1982). El Interaccionismo Simbólico. Perspectiva y método. Barcelona: Hora.

Ceirano, V. (2000). Las Representaciones Sociales de la Pobreza. Cinta moebio, 9, 337-350. http://www.moebio.uchile.cl/09/ceirano.html

De Alba, M. (2004). Mapas mentales de la Ciudad de México: una aproximación psicosocial al estudio de las representaciones espaciales. Estudios Demográficos y Urbanos, 55, 115-143. http://www.redalyc.org/pdf/312/31205503.pdf 
Debiec, J., \& Olsson, A. (2017). Social Fear Learning: from Animal Models to Human Function. Trends in Cognitive Sciences, 21(7), 546-555. http://dx.doi. org/10.1016/j.tics.2017.04.010

Diazgranados, P., \& Sánchez, D. (2014). Representación social del trabajo en jóvenes que culminan sus estudios de bachillerato en el distrito de Santa Marta. Psicogente, 17(32), 406-420. http://www.scielo.org.co/pdf/psico/v17n32/v17n32a12. pdf

Durkheim, E. (1996). Reglas del Método Sociológico. Madrid: Orbis.

Duveen, G. (2013). Prefácio. In: S. Moscovici (Ed.), Representações sociais: Investigações em psicologia social. Petrópolis, RJ: Vozes.

Farah, A. (2011). Attitude and Social Representation. Procedia-Social and Behavioral Sciences, 30, 1593-1597. https://doi.org/10.1016/j.sbspro.2011.10.309

Flores, R. (2008). Representaciones sociales del medio ambiente. Perfiles Educativos, 120, 33-62. http://www.scielo.org.mx/scielo.php?script=sci_arttext\&pid=S0185-26982008000200003

Flores, R. (2010). Medio ambiente y educación ambiental: representaciones sociales de los profesores en formación. Magis, Revista Internacional de Investigación en Educación, 2(4), 401-414. http://www.redalyc.org/articulo.oa?id=281021692011

Gómez, M., Chamorro, G., Obispo, K., Parra, Y., Paba, C. \& Rodríguez, U. (2011). Representación social del psicólogo en el área de la salud: Un estudio cualitativo en la Facultad de Ciencias de la Salud de la Universidad del Magdalena. Psicología desde el Caribe, 30(1), 91-122. http://rcientificas.uninorte.edu.co/index.php/ psicologia/article/view/4457/6904

González, P. (2001). Imágenes de Ciudad: Percepción y Cognición en niños de Bogotá. (Trabajo de Grado) Universidad de los Andes, Bogotá. https://books.google.com. br/books/about/Im\%C3\%A1genes_de_ciudad.html?id=RvpYAAAAMAAJ\&redir_ esc $=y$

Holahan, C. (2004). Psicología Ambiental, un enfoque general. México: Limusa. http://files.jovenes-eternamente.webnode.mx/200000043-598bd5a8e6/Psicologia\%20ambiental.pdf

Jodelet, D. (1984). La representación social: Fenómeno, Concepto y Teoría. En S. Moscovici (Ed.), Psicología Social II, Pensamiento y Vida Social, Psicología Social y Problemas Sociales. (pp.469-506) Buenos Aires: Paidós.

Jodelet, D. (2011). Conferência de Denise Jodelet por ocasião do recebimento do título de doutor Honoris Causa da Universidade Federal do Rio de Janeiro. In A. J. Mazzotti (Ed.), Representações sociais: aspectos teóricos e aplicações à educação. Múltiplas Leituras, 1(1), 18-43. file://C:/Users/usuario/Downloads/11691961-1-PB.pdf

Jodelet, D. (2015). Problématiques psychosociales de l'approche de la notion de sujet. Cadernos de Pesquisa, 45(156), 315-327. http://dx.doi. org/10.1590/198053143203

Jovchelovitch, S. (1998). Representações Sociais: para uma fenomenologia dos saberes sociais. Psicologia e Sociedade, 10(1), 54-68. http://eprints.Ise.ac.uk/2595/

Kaës, R. (1976). L'appareil psychique groupal: constructions du groupe. Paris: Dunod. http://pedagopsy.eu/livre_kaes_appareil.html

Knight, S., \& Littleton, K. (2015). Thinking, interthinking, and technological tools. In R. Wegerif, L. Li, \& J. C. Kaufman (Eds.), The Routledge international handbook of research on teaching thinking (p. Section 7(al). Londres, Reino Unido. Retrieved from http://www.routledge.com/books/details/9780415747493/ 
Knight, S., \& Littleton, K. (2017). Socialising Epistemic Cognition. Educational Research Review, 21, 17-32. http://dx.doi.org/10.1016/j.edurev.2017.02.003

Knight, S., Arastoopour, G., Williamson, D., Buckingham, S., \& Littleton, K. (2014). Epistemic networks for epistemic commitments. In International conference of the learning sciences. Boulder, CO: International Society of the Learning Sciences. Retrieved from http://oro.open.ac.uk/39254/.

Kuri-Pineda, E. (2017). La construcción social de la memoria en el espacio: una aproximación sociológica. Península, 12(1), 9-30. http://www.revistas.unam.mx/ index.php/peninsula/article/view/58261

Liberman, Z., Woodward, A., \& Kinzler, K. (2017). The Origins of Social Categorization. Trends in Cognitive Sciences, 21(7), 556-568. http://dx.doi.org/10.1016/j. tics.2017.04.004

López-Noguero, F. (2002). El análisis de contenido como método de investigación. Revista de Educación, 4, 167-179. http://rabida.uhu.es/dspace/bitstream/ handle/10272/1912/b15150434.pdf?sequen

Márquez-Pulido, E. (2014). Valor de uso y espacio urbano: la ciudad como eje central de la conformación política, cultural y simbólica de las sociedades. Revista Mexicana de Ciencias Políticas y Sociales, 59(222), 187-208. http://www.revistas. unam.mx/index.php/rmcpys/article/view/47729

Mendlovic-Pasol, B. (2014). ¿ Hacia una "nueva época" en los estudios de memoria social? Revista Mexicana de Ciencias Políticas y Sociales, 59(221), 291-316. http:// www.revistas.unam.mx/index.php/rmcpys/article/view/47710

Merlinsky, G. (2017). Cartografías do conflito ambiental na Argentina. Notas teórico-metodológicas. Acta Sociológica, 73 (May-August), 221-246. https://doi. org/10.1016/j.acso.2017.08.008

Ministerio de Salud (1993). Resolución 008430 por la cual se establecen las normas científicas, técnicas y administrativas para la investigación en salud. Santa Fe de Bogotá. https://www.minsalud.gov.co/sites/rid/Lists/BibliotecaDigital/RIDE/DE/ DIJ/RESOLUCION-8430-DE-1993.PDF

Mora, M. (2002). La teoría de las representaciones sociales de Serge Moscovici. Athenea Digital, 2(2), 1-25. http://atheneadigital.net/article/view/55

Moscovici, S. (1961). La psychanalyse, son image, son public. Paris: PUF.

Moscovici, S. (1963). Attitudes and opinions. Annual Review of Psychology, 14, 231-260. https://www.researchgate.net/publication/9631226_Attitudes_and_ Opinions

Moscovici, S. (1981). L'âge des foules. Un traité historique de psychologie des masses. Paris: Fayard. http://classiques.uqac.ca/contemporains/moscovici_serge/age_ des_foules/age_des_foules.html

Moscovici, S. (1984). La psychologie sociale. Paris: PUF.

Moscovici, S. (1990). The Origin of Social Representations: A Response to Michael. Neu'ldeas in Phychol, 8(3), 383-388. http://psycnet.apa.org/record/199115224-001

Moscovici, S. (1998). The history and actuality of social representations. In F. Uwe, The psychology of the social, New York: Cambridge University Press.

Moscovici, S. (2013). Representações sociais: Investigações em psicologia social. Petrópolis: Vozes.

Moscovici, S., \& Personnaz, B. (1980). Studies in Social Influence. V. Minority Influence and Conversion Behavior in a Perceptual Task. Journal of Experimental Social Psychology, 16, 270-282. https://doi.org/10.1016/0022-1031(80)90070-0 
Navarro, O. (2004). Representación Social del agua y de sus usos. Psicología desde el Caribe, (14), 222-236. http://www.redalyc.org/pdf/213/21301409.pdf

Navarro, O. (2006). Representación social del agua. Pre-til, 10(4), 72-97. http://rcientificas.uninorte.edu.co/index.php/psicologia/article/view/1785/5277

Navarro, O., \& Gaviria, N. (2010). Representaciones sociales del habitante de la calle. Univ. Psychol, 9(2), 345-355. http://revistas.javeriana.edu.co/index.php/ revPsycho/article/view/259

Pecly, R., \& Wachelke, J. (2013). Índices complementares para o estudo de uma representação social a partir de evocações livres: raridade, diversidade e comunidade. Revista Psicologia: Teoria e Prática, 15(2), 119-129. http://pepsic.bvsalud.org/ scielo.php?script=sci_arttext\&pid=S1516-36872013000200009

Piñuel, J. (2002). Epistemología, metodología y técnicas del análisis de contenido. Estudios de Sociolingüística, 3(1), 1-42. https://www.ucm.es/data/cont/ docs/268-2013-07-29-Pinuel_Raigada_AnalisisContenido_2002_EstudiosSociolinguisticaUVigo.pdf

Reigota, M. (1995). Meioambiente e Representação Social. São Paulo: Cortez.

Rodríguez, U. (2006). El impacto del hombre en la naturaleza: Una perspectiva desde la Psicología Ambiental y la Economía. Duazary, 3(1), 60-63. http://dx.doi. org/10.21676/2389783X.608

Rodríguez, U. (2009). Representación socio-espacial del Centro Histórico de la ciudad de Santa Marta. Aproximación al constructo de identidad de lugar. Duazary, 6(2), 102-111. http://dx.doi.org/10.21676/2389783X.675

Rodríguez, U. (2011). Representación social y análisis del Referido semántico del medioambiente y la educación ambiental en la Universidad del Magdalena. Duazary, 8(1), 24-33. http://dx.doi.org/10.21676/2389783X.248

Rodríguez, U., Cortés, S., \& Varela, M. (2006). Mapas mentales del Centro Histórico de Santa Marta. Duazary, 3(2), 153-163. http://dx.doi.org/10.21676/2389783X.620

Rodríguez, U., Espitia, J., Montenegro, W., Ortiz, F., \& Suárez, Y. (2014). Representación social de la memoria en una muestra universitaria de ingeniería de sistemas y antropología. Duazary, 11(1), 7-13. http://dx.doi.org/10.21676/2389783X.715

Rodríguez, U., \& Paba, C. (2013). Representación Social de la Crisis Ambiental. Psicogente, 16(29), 84-102. http://revistas.unisimon.edu.co/index.php/psicogente/ article/view/1943

Silva, C., Carmo, G., \& Silva, A. (2015). Breves observações sobre a teoria das representações sociais de Serge Moscovici e a interdisciplinaridade. Estudos Interdisciplinares em Psicologia, 6(2), 59-70. http://dx.doi.org/10.5433/22366407.2015v6n2p59

Suchow, J., Bourgin, D. \& Griffiths, T. (2017). Evolution in Mind: Evolutionary Dynamics, Cognitive Processes, and Bayesian Inference. Trends in Cognitive Sciences, 21(7), 522-530. http://dx.doi.org/10.1016/j.tics.2017.04.005

Touraine, A. (2007). Penser autrement. Paris: Fayard.

Zamora-Saenz, I. (2017). Constructivismo y realismo crítico en los conflictos ambientales. Acta Sociológica, 73 (May-August), 273-294. http://www.revistas.unam. $\mathrm{mx} /$ index.php/ras/article/view/61062

Zavalloni, M. (2007). Ego-écologie et identité: Une approche naturaliste. Paris: PUF. https://journals.openedition.org/osp/2368

Esta obra está bajo: Creative commons attribution 4.0 international license. El beneficiario de la licencia tiene el derecho de copiar, distribuir, exhibir y representar la obra y hacer obras derivadas siempre y cuando reconozca y cite la obra de la forma especificada por el (cc) BY 


\section{Anexo 1}

Instrumento para la técnica de Asociación Libre.

\section{Pregunta 1:}

Sin pensarlo mucho, qué palabras o frases se le ocurren cuando escucha el término "Centro Historico de Santa Marta"?.

\section{Pregunta 2:}

Si pensarlo Mucho, mencione 5 lugares que usted considere representativos del Centro Histórico de Santa Marta. Luego al frente de ellos, en el paréntesis, escriba en orden ascendente el número que establezca el orden de importancia que usted considere (ejem.: 1 el más importante, 5 el menos importante).

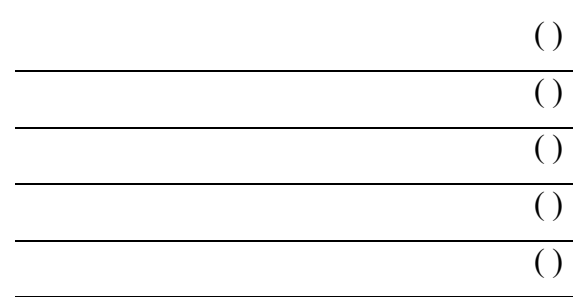

Sexo $M() F(~)$.

Edad :

Tiempo de vivir en Santa Marta (en años): 


\section{Anexo 2}

\section{Escala de Valoración Ambiental y Dimensión Afectiva.}

1. Según los criterios que aparecen debajo de cada lugar, valórelos en una escala de de 1 a 5 los lugares señalados. Ej: el valor de "1" es desorganizado y el valor " 5 " es Organizado.

\begin{tabular}{|c|c|c|c|c|c|c|c|c|c|c|c|c|c|}
\hline \multicolumn{7}{|c|}{ 1. Catedral } & \multicolumn{7}{|c|}{ 2. Plaza Simón Bolívar } \\
\hline \multicolumn{7}{|c|}{ VALORACIÓN } & \multicolumn{7}{|c|}{ VALORACIÓN } \\
\hline \multirow{12}{*}{$\begin{array}{r}\text { Desorganizado } \\
\text { Inseguro } \\
\text { No Incluyente } \\
\text { Sucio } \\
\text { Desordenado } \\
\text { No atractivo } \\
\text { Incómodo } \\
\text { Triste } \\
\text { Ruidoso } \\
\text { Intranquilo } \\
\text { Desagradable } \\
\text { Excitante }\end{array}$} & 1 & 2 & 3 & 4 & 5 & \multirow{12}{*}{$\begin{array}{l}\text { Organizado } \\
\text { Seguro } \\
\text { Incluyente } \\
\text { Limpio } \\
\text { Ordenado } \\
\text { Atractivo } \\
\text { Cómodo } \\
\text { Alegre } \\
\text { Silencioso } \\
\text { Tranquilo } \\
\text { Agradable } \\
\text { Relajante }\end{array}$} & \multirow{12}{*}{$\begin{array}{r}\text { Desorganizado } \\
\text { Inseguro } \\
\text { No Incluyente } \\
\text { Sucio } \\
\text { Desordenado } \\
\text { No atractivo } \\
\text { Incómodo } \\
\text { Triste } \\
\text { Ruidoso } \\
\text { Intranquilo } \\
\text { Desagradable } \\
\text { Excitante }\end{array}$} & \begin{tabular}{|l|l}
1 \\
\end{tabular} & 2 & 3 & 4 & 5 & \multirow{12}{*}{$\begin{array}{l}\text { Organizado } \\
\text { Seguro } \\
\text { Incluyente } \\
\text { Limpio } \\
\text { Ordenado } \\
\text { Atractivo } \\
\text { Cómodo } \\
\text { Alegre } \\
\text { Silencioso } \\
\text { Tranquilo } \\
\text { Agradable } \\
\text { Relajante }\end{array}$} \\
\hline & 1 & 2 & 3 & 4 & 5 & & & 1 & 2 & 3 & 4 & 5 & \\
\hline & 1 & 2 & 3 & 4 & 5 & & & 1 & 2 & 3 & 4 & 5 & \\
\hline & 1 & 2 & 3 & 4 & 5 & & & 1 & 2 & 3 & 4 & 5 & \\
\hline & 1 & 2 & 3 & 4 & 5 & & & 1 & 2 & 3 & 4 & 5 & \\
\hline & 1 & 2 & 3 & 4 & 5 & & & 1 & 2 & 3 & 4 & 5 & \\
\hline & 1 & 2 & 3 & 4 & 5 & & & 1 & 2 & 3 & 4 & 5 & \\
\hline & 1 & 2 & 3 & 4 & 5 & & & 1 & 2 & 3 & 4 & 5 & \\
\hline & 1 & 2 & 3 & 4 & 5 & & & 1 & 2 & 3 & 4 & 5 & \\
\hline & 1 & 2 & 3 & 4 & 5 & & & 1 & 2 & 3 & 4 & 5 & \\
\hline & 1 & 2 & 3 & 4 & 5 & & & 1 & 2 & 3 & 4 & 5 & \\
\hline & 1 & 2 & 3 & 4 & 5 & & & 1 & 2 & 3 & 4 & 5 & \\
\hline 3. Car & 1el & & & & & & 4. Mus & seo & de & $\mathrm{O}$ & & & \\
\hline & & $A L C$ & $\mathrm{RA}$ & IÓ & & & & & $A L C$ & RA & 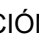 & & \\
\hline Desorganizado & 1 & 2 & 3 & 4 & 5 & Organizado & Desorganizado & \begin{tabular}{|l|}
1 \\
\end{tabular} & 2 & 3 & 4 & 5 & Organizado \\
\hline Inseguro & 1 & 2 & 3 & 4 & 5 & Seguro & Inseguro & 1 & 2 & 3 & 4 & 5 & Seguro \\
\hline No Incluyente & 1 & 2 & 3 & 4 & 5 & Incluyente & No Incluyente & 1 & 2 & 3 & 4 & 5 & Incluyente \\
\hline Sucio & 1 & 2 & 3 & 4 & 5 & Limpio & Sucio & 1 & 2 & 3 & 4 & 5 & Limpio \\
\hline Desordenado & 1 & 2 & 3 & 4 & 5 & Ordenado & Desordenado & 1 & 2 & 3 & 4 & 5 & Ordenado \\
\hline & 1 & 2 & 3 & 4 & 5 & Atractivo & No atractivo & 1 & 2 & 3 & 4 & 5 & Atractivo \\
\hline & 1 & 2 & 3 & 4 & 5 & Cómodo & Incómodo & 1 & 2 & 3 & 4 & 5 & Cómodo \\
\hline Triste & 1 & 2 & 3 & 4 & 5 & Alegre & Triste & 1 & 2 & 3 & 4 & 5 & Alegre \\
\hline & 1 & 2 & 3 & 4 & 5 & Silencioso & Ruidoso & 1 & 2 & 3 & 4 & 5 & Silencioso \\
\hline & 1 & 2 & 3 & 4 & 5 & Tranquilo & Intranquilo & \begin{tabular}{|l|}
1 \\
\end{tabular} & 2 & 3 & 4 & 5 & Tranquilo \\
\hline & 1 & 2 & 3 & 4 & 5 & Agradable & Desagradable & 1 & 2 & 3 & 4 & 5 & Agradable \\
\hline & 1 & 2 & 3 & 4 & 5 & Relajante & Excitante & \begin{tabular}{|l}
1 \\
\end{tabular} & 2 & 3 & 4 & 5 & Relajante \\
\hline 5. Claustro & $\mathrm{S}$ & $n$ & ua & 18 & $\mathrm{p}$ & uceno & & & & & & & \\
\hline & & $\mathrm{ALC}$ & $\mathrm{RA}$ & IÓ & & & & & & & & & \\
\hline Desorganizado & 1 & 2 & 3 & 4 & 5 & Organizado & & & & & & & \\
\hline Inseguro & 1 & 2 & 3 & 4 & 5 & Seguro & & & & & & & \\
\hline No Incluyente & 1 & 2 & 3 & 4 & 5 & Incluyente & & & & & & & \\
\hline Sucio & 1 & 2 & 3 & 4 & 5 & Limpio & & & & & & & \\
\hline Desordenado & 1 & 2 & 3 & 4 & 5 & Ordenado & & & & & & & \\
\hline No atractivo & 1 & 2 & 3 & 4 & 5 & Atractivo & & & & & & & \\
\hline Incómodo & 1 & 2 & 3 & 4 & 5 & Cómodo & & & & & & & \\
\hline Triste & 1 & 2 & 3 & 4 & 5 & Alegre & & & & & & & \\
\hline Ruidoso & 1 & 2 & 3 & 4 & 5 & Silencioso & & & & & & & \\
\hline Intranquilo & 1 & 2 & 3 & 4 & 5 & Tranquilo & & & & & & & \\
\hline Desagradable & 1 & 2 & 3 & 4 & 5 & Agradable & & & & & & & \\
\hline Excitante & 1 & 2 & 3 & 4 & 5 & Relajante & & & & & & & \\
\hline
\end{tabular}




\section{Anexo 3}

\section{Asociación Libre y Escala de Representación Social del Medio Ambiente}

Estamos haciendo una investigación sobre Representación Social del Medio Ambiente. Su participación es muy valiosa para nosotros. No existen respuestas correctas ni incorrectas, nos interesa conocer su experiencia en relación con el Medio Ambiente. Agradecemos su participación, su honestidad y claridad en las respuestas.

1. EDAD:

FECHA: $(\mathrm{D} / \mathrm{M} / \mathrm{A})$

2. GÉNERO: Masculino ( ). Femenino ( ).

3. ÁREA DE FORMACIÓN:

4. NIVEL DE FORMACIÓN (SEMESTRE):

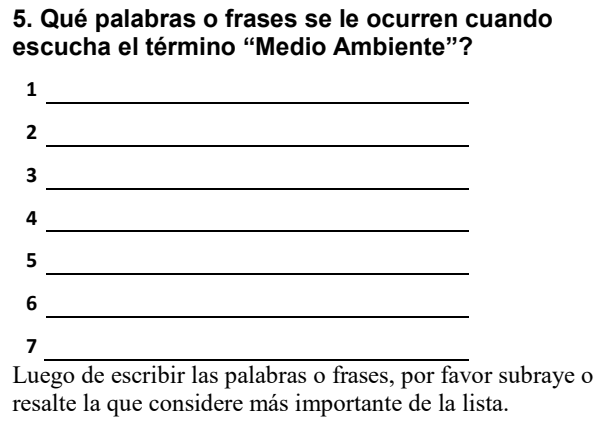

6. Que significa para usted Medio Ambiente?

7. Siente usted su vida amenazada por causa de problemas medio ambientales...

7.1. del barrio donde vive? $\square \mathrm{Si} \square \mathrm{No}$

7.2. de la ciudad donde vive? $\square \mathrm{Si} \quad \square$ No

7.3. del País donde vive? $\square \mathrm{Si} \square \mathrm{No}$

7.4. del Planeta donde vive? $\square \mathrm{Si} \quad \square \mathrm{No}$

\section{Cómo calificaría el Medio Ambiente?}

$$
\begin{array}{lll}
\text { Excelente } & \square 4 & \text { Malo } \\
\text { Bueno } & \square 5 & \text { Neutro } \\
\text { Regular } & &
\end{array}
$$

\section{Considera usted que....}

9.1. Su barrio está en crisis ambiental? $\square \mathrm{Si} \quad \square$ No 9.2. Su ciudad está en crisis ambiental? $\square \mathrm{Si} \quad \square$ No 9.3. Su País está en crisis ambiental? $\square \mathrm{Si} \quad \square \mathrm{No}$ 9.4. El Planeta está en crisis ambiental? $\square \mathrm{Si} \quad \square$ No
10. Ha experimentado problemas de salud por causas medio ambientales? $\square$ No

10.1. Qué tipo de Problema?

102. Quién es el responsable del problema?

......................

11. En su opinión a qué se refiere el Medio Ambiente?

(Puede escoger varias opciones)

\begin{tabular}{|l|l|}
\hline 1. A lo natural & \\
\hline 2. A lo construido por el hombre & \\
\hline 3. A la cultura & \\
\hline 4. A la historia & \\
\hline 5. Al hombre & \\
\hline 6. A los animales y los vegetales & \\
\hline 7. Otro & \\
\hline
\end{tabular}

12. A quien pertenece el Medio Ambiente?

(Puede escoger varias opciones)

1. A todas las personas

2. A la humanidad

3. Al gobierno, al Estado

4. A las Organizaciones protectoras del

ambiente

5. A todos los seres vivos

\section{Cuál es la causa de la Crisis Ambiental}

(Puede escoger varias opciones)

\begin{tabular}{|l|l|}
\hline 1. A todas las personas & \\
\hline 2. A la humanidad & \\
\hline 3. Al gobierno, al Estado & \\
\hline $\begin{array}{l}\text { 4. A las Organizaciones protectoras del } \\
\text { ambiente }\end{array}$ & \\
\hline 5. A todos los seres vivos & \\
\hline
\end{tabular}

